\title{
SHEAR VISCOSITY VIA PERIODIC NONEQUILIBRIUM MOLECULAR DYNAMICS
}

\author{
W.T. ASHURST \\ Sandia Livermore Laboratory, Livermore, Califomia 94550, USA \\ and \\ W.G. HOOVER \\ Department of Applied Science, UC Davis, Livermore, Califomia 94550, USA
}

Received 7 March 1977

\begin{abstract}
Nonequilibrium molecular dynamic results of shear viscosity near the freezing density have a strain rate dependence that is best described by the Ree--Eyring hyperbolic arcsine relation. A square root dependence is not consistent with low gradient results or theoretical predictions. We conclude that symmetry, experiment, calculation, and theory all favor the Ree-Eyring form.
\end{abstract}

Naitoh and Ono, by simulating Couette flow, recently measured the hardsphere viscosity coefficient for 108 hard spheres at the freezing density [1]. A periodic system undergoing homogeneous shear was studied with molecular dynamics. Similar calculations have also been carried out using the Lennard-Jones potential $[2-4]$.

At the freezing density Naitoh and Ono use a squareroot dependence of viscosity on strain rate to extrapolate their data to the zero-gradient limit of NavierStokes hydrodynamics. These authors state that the finite-gradient correction is in accord with the prediction of Kawasaki and Gunton [5]. Kawasaki and Gunton, however, actually predicted a dependence several orders of magnitude smaller. In fact, the data presented in fig. 1 of ref. [1] cover too small a range of strain rates to clearly support a square-root form. These data can be fitted equally well with the ReeEyring hyperbolic arcsine relation,

$\eta(\omega) / \eta(0)=\sinh ^{-1}(\omega \tau) /(\omega \tau)$

where $\omega$ is strain rate and $\tau$ is a relaxation time. Such a fit is shown in our own fig. 1 . The $\sinh ^{-1}$ function is smooth at the origin and also has been confirmed experimentally, for some materials, over many decades of strain rate [6]. Its use in fitting the hard-sphere data significantly reduces $\eta(0)$, from 11.7 to 9.4 in units of $(m k T) / \sigma^{2}$, and removes about half of the serious disagreement between the Naitoh-Ono work and the earlier equilibrium (Green-Kubo relations) calculations

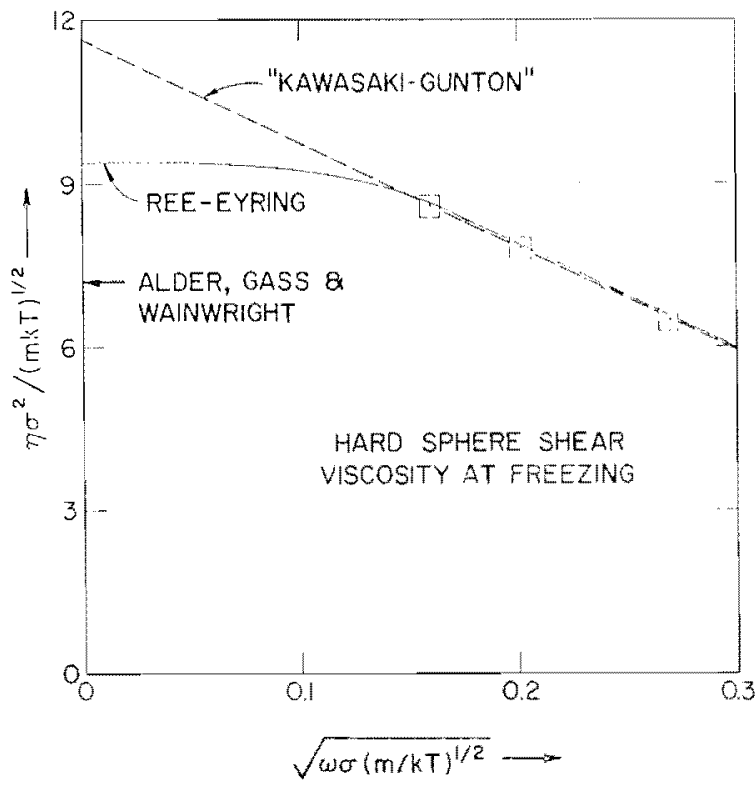

Fig. 1. Hars-sphere shear viscosity at freezing [1]. The data, from fig. 1 of ref. [1], are viscosities of $8.65,7.85$, and 6.55 $(m k T)^{1 / 2} / \sigma^{2}$, at strain rates of $0.0253,0.0408$, and 0.0724 $(\mathrm{kT} / \mathrm{m})^{1 / 2} / \sigma$. The straight-line intercept of 11.7 considerably exceeds the (solid-line) Ree-Eyring value of $9.4(m k T)^{1 / 2} / \sigma^{2}$. The Ree-Eyring extrapolation corresponds to a viscous rela* xation time of $30 \sigma(m / k T)^{1 / 2}$. The 500-particle Alder-Gass... Wainwright viscosity is $7.2(m k T)^{1 / 2} / \sigma^{2}$.

[7] of Alder et al. The required relaxation time $\tau$ is of the same order as the time required to diffuse one inter- 


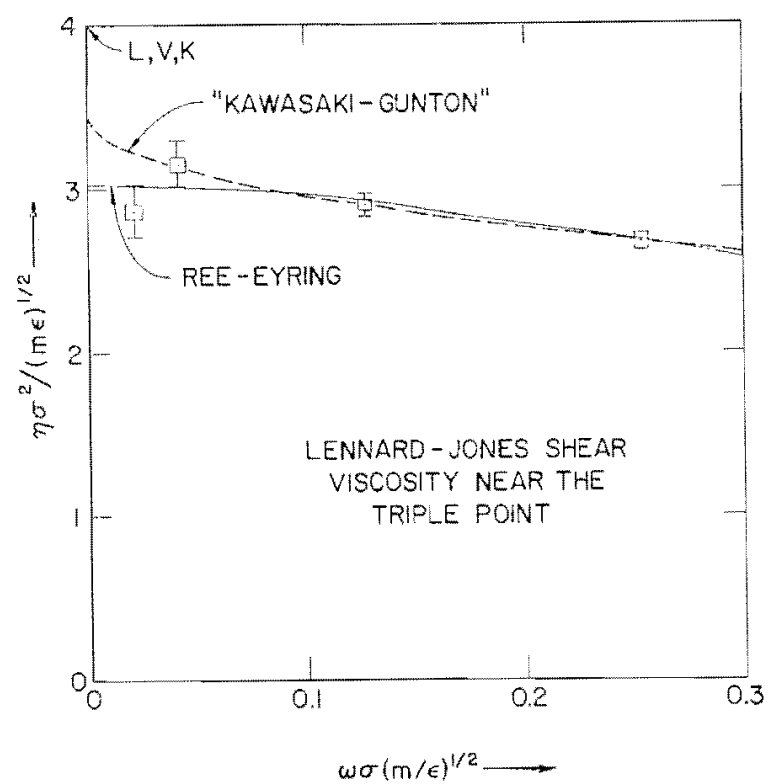

Fig. 2. Lennard-Jones shear viscosity near the triple point as a function of strain rate. The data appear in table 1 . While the larger-strain-rate data can be described by the Kawasaki-Gunton square-root dependence (dashed-line), the Ree-Eyring hyperbolic aresine (solid-line) describes the data better and agrees well with the experimental viscosity for argon, $3.0 \pm 0.06(m e)^{1 / 2} / 0^{2}$.

particle spacing in the fluid, $\tau \sim(V / N)^{2 / 3 / 6 D}$. The hard-sphere relaxation time we find in fig. 1 is about three times that which would be estimated by scaling our Lennard-Jones triple point calculations.

The Lennard-Jones relaxation time is itself not well tied down because it varies with the particular choice of molecular dynamic method. Our previous Couette flow results produced a time of $8.7 \mathrm{\sigma}(\mathrm{m} / \mathrm{\epsilon})^{1 / 2}$ for flow driven by boundary regions $[3]$ and a considerably smaller time, $5.5 \mathrm{o}(\mathrm{m} / \epsilon)^{1 / 2}$, for a periodic nonequilibrium system with an external force applied to each particle each time step to produce the desired velocity gradient [4]. New calculations have been done using Naitoh and Ono's method (displaced images with no external forces) but with a small particle-velocity reduction each time step, as needed, to maintain a constant temperature. We find no noticeable width dependence when this velocity reduction is carried out with respect to the local mean velocity (see table 1). The zero-gradient value from a hyperbolic arcsine fit agrees with our previous results (and
Table 1

Shear viscosities from periodic nonequilibrium molecular dynamics near the Lennard-Jones triple point. Dependence on system width (one and two 108-particle cubes) and strain ratea.

\begin{tabular}{|c|c|c|c|c|}
\hline \multirow[b]{2}{*}{$N^{\prime \prime}$} & \multirow[b]{2}{*}{$\omega \sigma(m / \epsilon)^{1 / 2}$} & \multicolumn{3}{|l|}{$\eta \sigma^{2} /(m \varepsilon)^{1 / 2}$} \\
\hline & & Total & Kinetic & Time \\
\hline 216 & 0.0217 & $2.87 \pm 0.17$ & 0.060 & 60 \\
\hline 216 & 0.0422 & $3.17 \pm 0.14$ & 0.087 & 64 \\
\hline 108 & 0.0418 & $3.15 \pm 0.17$ & 0.073 & 48 \\
\hline 216 & 0.127 & $2.87 \pm 0.056$ & 0.074 & 96 \\
\hline 108 & 0.127 & $2.89 \pm 0.11$ & 0.072 & 96 \\
\hline 108 & 0.253 & $2.67 \pm 0.027$ & 0.081 & 144 \\
\hline
\end{tabular}

a The reduced density $\mathrm{No}^{3} / \mathrm{V}$ is 0.8442 and the temperature $k T / \epsilon$ was maintained at 0.722 by rescaling particle velocities at each time step with respect to the desired velocity gradient. The average reduction in particle kinetic energy was 0.44 percent at the largest gradient. The observation time is given in terms of relative displacement of the boundary surface (in units of the 108-particle cube edge).

with experimental data for argon). The relaxation time using this method is even smaller, $4.0 \sigma(\mathrm{m} / \epsilon)^{1 / 2}$.

A considerably larger zero-gradient viscosity would be predicted if the square-root gradient dependence were fitted to the three largest-gradient results, as is shown in fig. 2 (dashed line).

We have also studied strain-rate effects at a somewhat lower density using the purely-repulsive part of the Lennard-Jones potential (cut off at the energy minimum). This system was previously studied to examine the long-time tail effect for self-diffusion [8]. At a reduced density of $\mathrm{No}^{3} / \mathrm{V}=0.45$ and a reduced temperature of $k T / \epsilon=2.16$ the previous Couette flow result was $\eta \sigma^{2} /(m \epsilon)^{1 / 2}=0.45 \pm 0.03$. The new result is $0.47 \pm 0.03$. The kinetic contributions to these reduced viscosities are respectively 0.21 and 0.18 . At this low density, about half the freezing density, neither method indicates a width or strainrate dependence.

In the soft-potential results presented here there still remain some relatively minor discrepancies between the freezing density viscosities calculated by alternative methods. The equilibrium results of Levesque et al. [9] $\left(4.0 \pm 0.3(m \epsilon)^{1 / 2} / \sigma^{2}\right)$ is consider* ably above our prediction for the infinite-system zero strain rate value: $2.9 \pm 0.1(m \epsilon)^{1 / 2} / 0^{2}$. Applying our width correction to the Levesque et al. result produces 
a value of $3.15 \pm 0.3$, which does agree with our nonequilibrium results (three different methods) and the experimental argon value $\left(3.0 \pm 0.06(m \epsilon)^{1 / 2} / 0^{2}\right)$. The number-dependence of Green-Kubo calculations should be investigated. For the hard spheres at freezing the situation is worse. The nonequilibrium and equilibrium methods still are in marked disagreement, even using the $\sinh ^{-1}$ relation, and the freezing-point number dependence has not been studied with either method.

\section{References}

[1] T. Naitoh and S. Ono, Physics Letters A 57A (1976) 448.
[2] A.W. Lees and S.F. Edwards, J. Phys. C: Solid State Phys. 5 (1972) 1921.

[3] W.T. Ashurst and W.G. Hoover, Phys. Rev. A11 (1975) 658.

[4] W.G. Hoover and W.T. Ashurst, Theoretical chemistry, Advances and perspectives 1, eds. H. Eyring and D. Henderson (Academic Press, 1975) p. 1.

[5] K. Kawasaki and J. Gunton, Phys. Rev. A8 (1973) 2048.

[6] F.H. Ree, T. Ree and H. Eyring, Ind. Eng. Chem. 50 (1958) 1036.

[7] B.J. Alder, D.M. Gass and T.E. Wainwright, J. Chem. Phys. $53(1970) 3813$.

[8] D. Levesque and W.T. Ashurst, Phys. Rev. Lett. 33 (1974) 277.

[9] D. Levesque, L. Verlet and I. Kurkijarvi, Phys. Rev, A7 (1973) 1690. 